\title{
Cost-utility analysis of imrecoxib compared with celecoxib for patients with osteoarthritis
}

\author{
Xueshan Sun ${ }^{1}$, Xuemei Zhen ${ }^{2,3}$, Xiaoqian $\mathrm{Hu}^{1}$, Yuanyuan $\mathrm{Li}^{1}$, Shuyan $\mathrm{Gu}^{4}$, Yuxuan $\mathrm{Gu}^{1}$, Zixuan $\mathrm{Zhao}{ }^{1}$, \\ Wei Yang ${ }^{5}$, Hengjin Dong ${ }^{1,6}$ \\ ${ }^{1}$ Center for Health Policy Studies, School of Public Health, Zhejiang University School of Medicine, Hangzhou, China; ${ }^{2}$ School of Health Care \\ Management, Shandong University, Jinan, China; ${ }^{3}$ NHC Key Laboratory of Health Economics and Policy Research (Shandong University), Jinan, \\ China; ${ }^{4}$ Center for Health Policy and Management Studies, School of Government, Nanjing University, Nanjing, China; ${ }^{5}$ Shanghai Su-Value Health \\ Scientific Ltd., Shanghai, China; ${ }^{6}$ The Fourth Affiliated Hospital Zhejiang University School of Medicine, Yiwu, China \\ Contributions: (I) Conception and design: X Sun, X Zhen, H Dong; (II) Administrative support: H Dong; (III) Provision of study materials or patients: \\ X Hu, S Gu; (IV) Collection and assembly of data: X Sun, Y Gu, Z Zhao; (V) Data analysis and interpretation: X Sun, W Yang; (VI) Manuscript \\ writing: All authors; (VII) Final approval of manuscript: All authors. \\ Correspondence to: Hengjin Dong, MD, PhD. Zijingang Campus, Zhejiang University, Rd 866 Yuhang, Xihu District, Hangzhou, Zhejiang, China; \\ The Fourth Affiliated Hospital Zhejiang University School of Medicine, Yiwu, China. Email: donghj@zju.edu.cn.
}

Background: The objective of this study is to compare the long-term cost-utility of imrecoxib and celecoxib for patients with osteoarthritis (OA) from the perspective of the Chinese healthcare system.

Methods: An economic model was built based on the model from the National Institute for Health and Care Excellence (NICE). The simulation was carried out initially for 100 cycles of 3 months each, starting with 10,000 patients. A discount rate of 5\% was applied both for cost and utility. Quality-adjusted life years (QALYs) were adopted as the utility indicator, and real-world data from the hospital information systems of 170 hospitals was collected to indicate cost. The relative incidence rates of adverse events (AEs) with imrecoxib and celecoxib were collected from randomized controlled trials. Sensitivity analysis was performed to validate the robustness of the model.

Results: In the base case analysis (6-month treatment duration, 55 years old and above), imrecoxib was the more cost-effective option compared to celecoxib, with an incremental cost-effectiveness ratio (ICER) of $\$ 3,041.14$. This finding remained unchanged after varying the treatment duration and the age of the patients. The main drivers of the results were the relative incidence of myocardial infarction (MI), the cost of imrecoxib, and the utility of OA patients without any AEs. Probability sensitivity analysis (PSA) showed that there was a $59.02 \%$ probability of imrecoxib as the more cost-effective option, with a threshold of $\$ 30,000$.

Conclusions: Although there were uncertainties, imrecoxib was the more cost-effective option compared to celecoxib, with a definite possibility. Due to the limitations of the original model and this study, the results of this study should be adopted with caution.

Keywords: Imrecoxib; celecoxib; cost-utility; osteoarthritis (OA)

Submitted Dec 23, 2020. Accepted for publication Mar 29, 2021.

doi: $10.21037 / \mathrm{atm}-21-290$

View this article at: http://dx.doi.org/10.21037/atm-21-290

\section{Introduction}

Arthritis is listed as a type of 'immortal cancer' by the World Health Organization (WHO), and osteoarthritis $(\mathrm{OA})$ is the most common arthritic disease $(1,2)$. OA is a chronic degenerative disease characterized by joint pain throughout the body, which may be caused by fibrosis, chap, ulcers, and loss of articular cartilage (3). The prevalence of OA in China warrants close attention, as it reached $25.03 \%$, $21.51 \%, 20.46 \%$, and $8.99 \%$ for the lumbar, knee, cervical, and hand joints, respectively (4). In addition, as a chronic 
joint disease, it is especially harmful to the health of the elderly, and the prevalence of OA increases with age (5). China entered into an aging society in 1999 (6). Given the age-related prevalence of $\mathrm{OA}$ and the growing number of elderly people in China, the number of patients with OA in China will increase year by year. Furthermore, OA is a disease with a high disability rate, and can increase the incidence of cardiovascular diseases (CV) and all-cause mortality. For example, symptomatic OA of the knee can increase all-cause mortality by nearly $100 \%$ (3). According to the Chinese Guidelines for Diagnosis and Treatment of Osteoarthritis, there are six main types of drugs for managing osteoarthritis: Nonsteroidal anti-inflammatory drugs (NSAIDs), analgesics, intraarticular injection of drugs, disease-modifying antirheumatic drugs (DMARDs), Chinese patent medicine and antidepressants (3). Among the six types, NSAIDs are first-line used drugs, and widely prescribed to patients with OA. The anti-inflammatory mechanism of NSAIDs is to inhibit cyclooxygenase (COX), which is required for prostaglandin synthesis (7). COX exists in 2 isoforms: COX-1 is a ubiquitous constitutive isozyme producing prostaglandins, and is responsible for homeostatic functions, while COX-2 is largely a cytokineinduced isozyme producing prostaglandins that mediates pain and inflammation. There is a known link between NSAID therapy and gastrointestinal (GI) bleeding, with a reported 3,200 deaths in the US a year as a result of complications from GI bleeding using NSAIDs (8). Because COX-1 exists in the stomach, intestine, kidneys and platelets, and COX-2 is expressed during inflammation, the therapeutic effects of NSAIDs are mainly the result of inhibition of COX-2, whereas the toxic effects (e.g., gastrointestinal) are mainly due to the inhibition of COX-1 (9). Therefore, an ideal NSAID should selectively inhibit COX-2 without inhibiting COX-1 (7). COX2 inhibitors include: etodolac, meloxicam, celecoxib, rofecoxib, etoricoxib, valdecoxib and lumiracoxib, among which celecoxib is a common COX-2 inhibitor for managing $\mathrm{OA}$ and widely prescribed for managing $\mathrm{OA}$ (1). Imrecoxib, approved by the Chinese Food and Drug Administration (CFDA) in 2011, is a novel and moderately selective COX-2 inhibitor that possesses anti-inflammatory effects by inhibiting COX-2 (10,11).

Several studies have reported similar efficacy between celecoxib and imrecoxib, though with different prices and incidences of adverse events (AEs) (12-14). A review of the literature showed that there is a lack of costeffectiveness analyses on imrecoxib and celecoxib, causing great confusion for patients, hospitals, medical insurance departments, and policy makers. Meanwhile, the National Institute for Health and Care Excellence (NICE) developed an economic model for the cost-effectiveness analysis of drugs treatments for OA in 2008, which has been adopted in different countries $(8,15,16)$. The OA model was built based on the adverse events occurred due to the drugs used for osteoarthritis, which were also common adverse events in Chinese population. Therefore, the objective of this study is to compare the cost-effectiveness of imrecoxib and celecoxib for the treatment of OA based on the NICE OA model, with an update including Chinese real-world data from the perspective of the Chinese healthcare system. It is hoped that these results can be a reference for both related stakeholders in China and international experts who may be interested in the cost-effectiveness of different treatments for OA.

We present the following article in accordance with the CHEERS reporting checklist (available at http://dx.doi. org/10.21037/atm-21-290).

\section{Methods}

The model built in this study was an economic model for OA from the NICE, which was first built in the 2008 OA guidelines (guideline code: CG59), and was updated with data from the CONDOR trial in $2012(16,17)$. In the present study, the perspective was from the Chinese healthcare system, and the comparators were imrecoxib and celecoxib.

\section{Model structure}

The model was a combination of a Markov model and a decision tree model, with the aim to explore the costeffectiveness of 2 drug treatments for OA. The health states in this model were divided by the most frequent AEs caused by different drug treatments. There were 15 health states in this model: (I) 7 initial health states according to the main AEs caused by most NSAIDs, including OA without any AEs, GI discomfort (dyspepsia), symptomatic ulcer, complicated GI events, myocardial infarction (MI), stroke, and heart failure (HF); (II) 5 post-AE health states (as GI discomfort was assumed to be a mild AE which could be cured without any post-treatment), including post symptomatic ulcer, post complicated GI events, post MI, post stroke, and post HF; (III) 1 post-treatment state, where after treatment, patients without any AEs and with 
GI events transitioned to the post-treatment state; (IV) 1 withdraw state, where patients withdrew due to GI events; (V) 1 absorbed state, and the dead state was applied as the absorbed state.

To start with, it was supposed that all patients were in the state of "OA without any AEs", and in each cycle, patients transitioned to the $6 \mathrm{AE}$ health states, then from the $\mathrm{AE}$ health states to the post-AE states. Patients in the state of "GI discomforts" were seen as not stopping the drug treatment, therefore, there was a possibility for patients to transition from "GI discomforts" to another AE state. After treatment, patients transitioned to the post state and would stay in the post state until death (patients without any AEs and GI discomforts transitioned to the post treatment state, and patients with AEs transitioned to the corresponding post-AE states). There was a possibility for all patients to die in each state in each cycle. For patients taking the medications, there was possibility for them to withdraw due to GI events. When patients stopped taking imrecoxib or celecoxib, the topical diclofenac solution was assumed to be adopted as a medication to manage OA.

\section{Model running parameters}

The time horizon of this model was a lifetime, and the model was terminated when patients reached 80 years old or death. In the base case analysis, the age of patients was set to 55 years old. The simulation was carried out initially for 100 cycles with 3 months in each cycle. Cohort simulation with 10,000 patients per cycle was performed. The annual discount rate of $5 \%$ was applied according to the Chinese Guidelines for Pharmacoeconomics Evaluations (18). Scenario analyses were adopted for different start-ages (65 years old) and different treatment durations (6, 12, 24 months treatment).

\section{Inputs}

The model had 3 key parameters, which were also the inputs: cost, utility, and transition probability (Table 1). Both qualitative and quantitative analyses were applied to collect the input data. Half-cycle corrections were applied when it came to cost and utility.

\section{Utility values}

Quality-adjusted life years (QALYs) were adopted as the utility values. Utility values for the health states were extracted from the NICE OA model (17). According to the guidelines, for OA without any AEs, the utility values depend on the utility of OA itself and the efficacy of different treatment strategies. The Western Ontario and McMaster Universities Osteoarthritis Index (WOMAC) is widely used to assess the severity level of disease, and can also reflect the efficacy of drugs indirectly, which can be converted to EQ-5D scores using the transfer to utility (TTU) technique. After reviewing, the NICE OA model supposed that the difference in efficacy between the NSAIDs/COX-2 drugs was not significant, therefore, the efficacies of imrecoxib and celecoxib included in this study were equal (17). Considering the suggestions from the guideline development group, after 3 months, the patients with $\mathrm{HF}$ were reverted to the no-complications utility score $(8,17)$.

\section{Update with Chinese costs}

Cost data in the NICE OA model was based on UK medical expenses, and in this study, cost was updated with Chinese costs after collecting real-world data and relevant literature. The real-world data was extracted from the $\mathrm{Su}-$ Value database (Shanghai Su-Value Health Scientific Ltd., Shanghai, China) (21). The Su-Value database encompasses information from the hospital information systems (HIS) of 170 hospitals in 20 provinces across China. Based on the ICD-10 categories, patients with OA, GI discomfort, symptomatic ulcer, complicated GI events, stroke, MI, and HF were recognized, and diagnostic information, medical expenses, and basic social characteristics were extracted from 2016 to 2018. For the state of "OA patients without any AEs", cost was calculated by drug cost and other outpatient expenses extracted from the Su-Value database, and drug cost was extracted from the Beijing Medicine Sunshine Purchasing system (14) and adjusted by the recommended dosage in each cycle by the doctors. For the state of patients with 6 initial AEs, the costs were calculated directly from the $\mathrm{Su}$-Value database. There was no maintenance cost, however, there was a higher risk of suffering from GI events for patients in the post complicated GI events state and the post symptomatic ulcer state according to the NICE OA model. Therefore, costs for post complicated GI events and post symptomatic ulcer were obtained by combining the recurrence rates and the cost of corresponding initial $\mathrm{AE}$ states (17). Maintenance costs were considered for post $\mathrm{CV}$ states, and the cost of post $\mathrm{CV}$ was extracted from the literature which reported the cost of Chinese patients. For 
Table 1 Model inputs

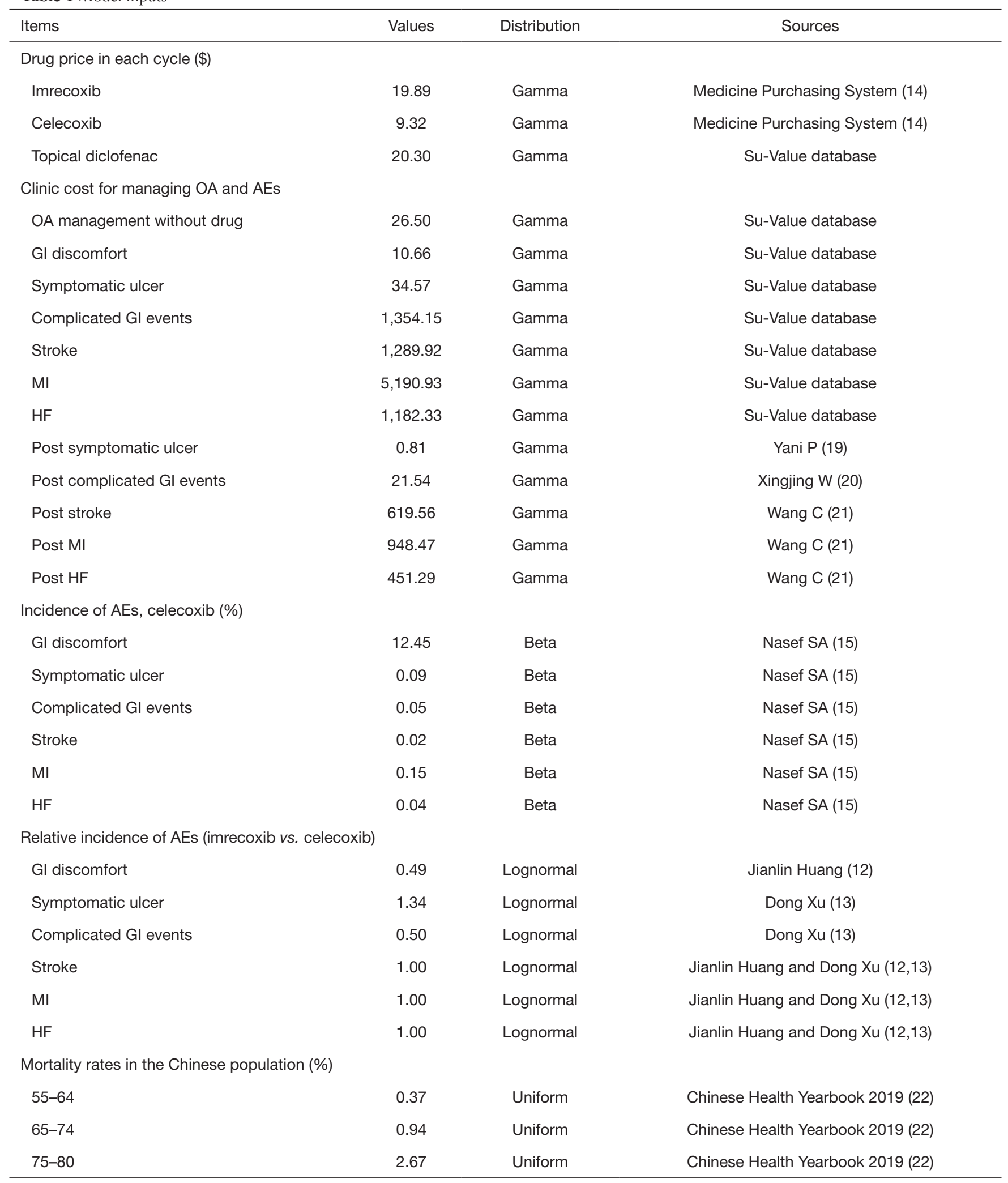

Table 1 (continued) 
Table 1 (continued)

\begin{tabular}{|c|c|c|c|}
\hline Items & Values & Distribution & Sources \\
\hline Gl discomforts & 0.733 & Lognormal & NICE OA model (17) \\
\hline Withdraw due to $\mathrm{Gl}$ & 0.989 & Lognormal & NICE OA model (17) \\
\hline Symptomatic ulcer & 0.552 & Lognormal & NICE OA model (17) \\
\hline Stroke & 0.348 & Lognormal & NICE OA model (17) \\
\hline MI & 0.374 & Lognormal & NICE OA model (17) \\
\hline $\mathrm{HF}$ & 0.710 & Lognormal & NICE OA model (17) \\
\hline Post symptomatic ulcer & 0.980 & Lognormal & NICE OA model (17) \\
\hline Post MI & 0.880 & Lognormal & NICE OA model (17) \\
\hline Post HF & 1.000 & Lognormal & NICE OA model (17) \\
\hline Post treatment & 0.989 & Lognormal & NICE OA model (17) \\
\hline
\end{tabular}

The maintenance costs of symptomatic ulcer and complicated Gl events were calculated by multiplying the cost of the initial state by the recurrence rate. The recurrence rates were $2.33 \%$ (23) and $1.59 \%$ (24) for symptomatic ulcer and complicated Gl events in each cycle, respectively. OA, osteoarthritis; AEs, adverse events; GI, gastrointestinal; MI, myocardial infarction; HF, heart failure; NICE, National Institute for Health and Care Excellence.

patients taking topical diclofenac to manage OA due to AEs, the cost of topical diclofenac was considered. The RMB exchange rate against the USD was 100:689.85 in 2019 (25), and all costs were adjusted to 2019 based on the exchange rate.

\section{Update with Chinese transition probabilities}

The incidence of AEs with celecoxib was extracted from the NICE OA model. Because imrecoxib is a new drug which was not included in the guidelines, the relative incidences of AEs with imrecoxib and celecoxib were extracted from the literature. The doses for celecoxib and imrecoxib were reported to be $200 \mathrm{mg}$ once a day (200 mg QD) and $100 \mathrm{mg}$ two times a day (100 mg BID) (14), respectively. The incidence rates of AEs of the drugs were adjusted, and it was assumed that the $\mathrm{AE}$ rate reduction had a relative incidence of half the dose reduction (16). The mortality rates were updated with data from the Chinese population. The observation period of the rate in the literature might not have been completely consistent with the period divided in the model, therefore, the probability was obtained by adjusting the instantaneous rate, and the formula was as follows: $r=-[\operatorname{In}(1-\mathrm{P} 1)] / t 1, \mathrm{P} 2=1-\exp (-\mathrm{rt} 2)$, where $\mathrm{r}$ represents the instantaneous rate, $\mathrm{P} 1$ represents the rate observed in the literature during the specific period, P2 is the probability needed in the model, $\mathrm{t} 1$ is the time of observation in the literature, and $t 2$ is the time of each cycle set in the study ( $\mathrm{t} 2=3$ months in this study) (26).

\section{Statistical analysis}

In the base-case analysis, the simulation was carried out for 100 cycles with 3 months in each cycle. Cohort simulation with 10,000 patients per cycle was performed. In the sensitivity analysis, both deterministic sensitivity analysis (DSA) and probability sensitivity analysis (PSA) were performed to validate the robustness of the model. In the DSA, discount rate was performed according to its preset range: $0-8 \%(18)$. Cost, utility, and transition probabilities were set up $\pm 50 \%$ for sensitivity analysis. In order to study the robustness of model outputs when multiple research variables changed simultaneously according to their corresponding distributions, PSA was 
Table 2 Incremental cost-effectiveness ratio

\begin{tabular}{lcc}
\hline Items & Imrecoxib & Celecoxib \\
\hline Total costs $(\$)$ & $1,169.40$ & $1,116.34$ \\
Total QALYs & 5.62 & 5.60 \\
Cost per QALY gained (imrecoxib vs. celecoxib) & & $3,041.14$ \\
\hline
\end{tabular}

QALYs, quality-adjusted life years.

carried out using a Monte Carlo approach with 10,000 iterations (16). Beta distribution was applied for utilities and transition probabilities, gamma distribution was applied for costs, lognormal distribution was applied for relative risk, and uniform distribution was applied for mortality rate. Estimates for which distributional information was unavailable were assumed to have lower and upper bounds of $95 \%$ confidence intervals equal to $50-150 \%$ of base case values (27) (Table S1).

TreeAge Pro Healthcare software was used to build and analysis the simulation model.

The authors are accountable for all aspects of the work in ensuring that questions related to the accuracy or integrity of any part of the work are appropriately investigated and resolved. This study was approved by the institutional review board of Zhejiang University School of Public Health (No. 20180923). Participants were given informed consent before taking part. All procedures performed in this study involving human participants were in accordance with the Declaration of Helsinki (as revised in 2013).

\section{Results}

\section{Incremental cost-effectiveness ratio (ICER)}

In the base case analysis, a 6-month treatment duration and patients aged 55 years old and above were applied. A lifetime time horizon was adopted, and it was demonstrated that treatment with imrecoxib resulted in costs of $\$ 1,169.40$ and benefits of 5.62 QALYs, while celecoxib resulted in costs of $\$ 1,116.34$ and benefits of 5.60 QALYs. Compared to treatment with celecoxib, treatment with imrecoxib was associated with an increase in costs of $\$ 53.06$ and an increase in benefits of 0.02 QALYs, resulting in an ICER of $\$ 3,041.14$ (Table 2).

\section{Parameters influencing the ICER}

After performing DSA, the results showed that the relative risk of MI, the cost of imrecoxib, and the utility of OA patients without any AEs were the main factors influencing the ICER of imrecoxib compared with celecoxib (Figure S1). All ICERs of imrecoxib $v s$. celecoxib were below $\$ 25,000$, and most of them were below $\$ 10,000$ (Figure S1). A further one-way sensitivity analysis was carried out, varying drug costs and relative incidence rates of AEs with imrecoxib and celecoxib (Figures 1,2). Different scenarios were carried out by varying the start age from 55 to 65 years old, and treatment duration from 6 to 24 months. It was found that ICERs were similar to the base case results, and the ICERs of imrecoxib vs. celecoxib varied from $\$ 2,814.97$ to $\$ 3,918.35$ (Table 3).

\section{Probabilistic representation of uncertainty}

PSA showed that there were more plots to the right of the threshold of \$30,000 (Figure 3). The cost-effectiveness acceptability curve also demonstrated the dominance of imrecoxib, as there was a $59.87 \%$ and $59.02 \%$ probability of imrecoxib as the more cost-effective drug, at a willingnessto-pay (WTP) of $\$ 10,000$ and $\$ 30,000$ per QALY, respectively (Figure 4).

\section{Discussion}

This analysis assessed the cost-effectiveness of imrecoxib and celecoxib based on the OA model built by the NICE, and an update with Chinese real-world data. Although there was a higher cost of treatment with imrecoxib, a gain in benefit was found when compared to treatment with celecoxib. Treatment with imrecoxib was supposed to be a more cost-effective option compared with celecoxib with the ICER of imrecoxib compared with celecoxib far lower than 1.0 GDP. The relative risk of MI, the cost of imrecoxib, and the utility of OA patients without any AEs were the main drivers of the results of the ICER, which was similar to Brereton et al.'s findings (8), while the main driver in the NICE model was the risk of stroke (16). The increased 


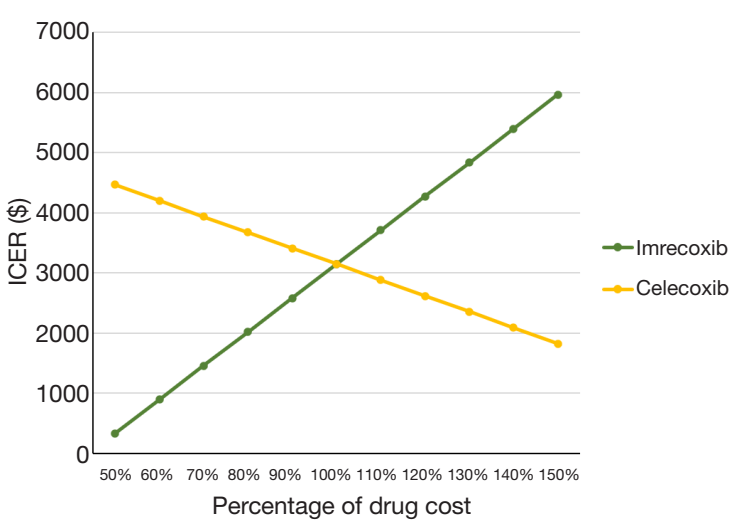

Figure 1 Sensitivity analysis varying drug cost. ICER, incremental cost-effectiveness ratio.

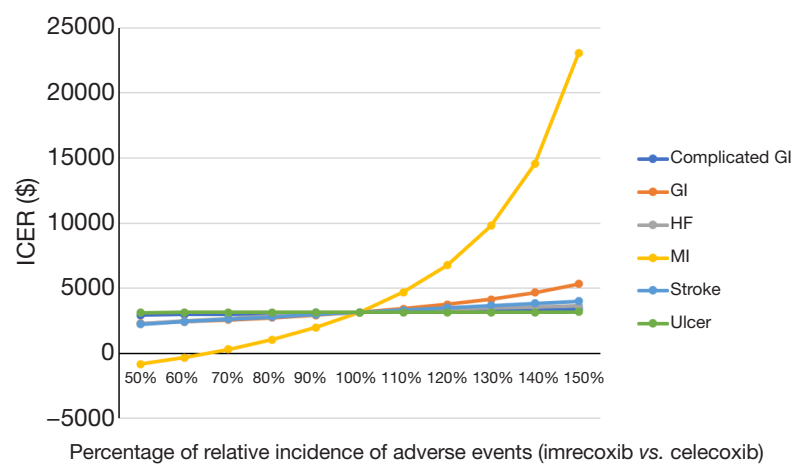

Figure 2 Sensitivity analysis varying the relative incidence of adverse events. ICER, incremental cost-effectiveness ratio; GI, gastrointestinal; HF, heart failure; MI, myocardial infarction; ICER, incremental cost-effectiveness ratio.

cost of imrecoxib drives the advantage of treatment with celecoxib, and vice versa. After varying the costs of the 2 drugs, the result was unchanged in that imrecoxib was the more cost-effective drug. In regards to the relative incidence of AEs with imrecoxib and celecoxib, the relative incidence of complicated GI events, ulcer, GI discomfort, stroke, and HF did not affect the results significantly, while the relative incidence of MI did affect the results significantly. In most cases, the results stayed robust, and all the ICERs were below $\$ 30,000$. The decrease in the relative incidence of $\mathrm{MI}$ favored treatment with imrecoxib. In addition to DSA, PSA was also performed to validate the robustness of the model, which is part of the reference case for technology submissions to the NICE $(26,28)$. The results of PSA also showed that imrecoxib was the more cost-effective drug when the parameters varied according to preset distributions, with the threshold of $\$ 10,000$. This was lower than the WHO-recommended ICER acceptability threshold of 1-time per capita GDP (which was \$10,276.44 in 2019) (25). The results of PSA validate the conclusions of this study, and should be paid attention to when adopting the conclusions.

Imrecoxib was first developed in 1997, and during development, the withdrawal of rofecoxib reminded developers that the value of $\mathrm{IC}_{50} \mathrm{COX}-1 / \mathrm{IC}_{50} \mathrm{COX}-2$ should remain under control (10). Because a higher risk would have been brought by over-inhibition, moderate inhibition was advised, and the value of $\mathrm{IC}_{50} \mathrm{COX}-1 / \mathrm{IC}_{50} \mathrm{COX}$ 2 was limited to $2-30$ (10). In addition to safety, similar efficacy to celecoxib was also required when developing the new COX-2 inhibitor. From the perspectives of both safety and efficacy, imrecoxib was finally selected among several choices. The value of $\mathrm{IC}_{50} \mathrm{COX}-1 / \mathrm{IC}_{50} \mathrm{COX}-2$ of imrecoxib was 6.39 , which was $77 \%$ of that of celecoxib, demonstrating that imrecoxib was more likely to have a lower incidence of AEs compared with celecoxib $(11,29)$, which could have also accounted for the lower relative incidence of several AEs adopted in this study. Nowadays, according to the list of national basic insurance drugs, imrecoxib is a second-line drug for managing OA (30), which challenges its wider application. Although there were uncertainties in the results of this study, considering the long-term cost-effectiveness of imrecoxib as a definite possibility, relevant departments can also take imrecoxib into consideration.

As with other economic models, as well as the original NICE OA model, there are some limitations. First, the relative incidences of several AEs of different treatments were extracted from randomized controlled trials (RCTs), and there was a lack of pooling of observational data, which was also a limitation in the original model. Given this uncertainty, sensitivity analysis varying the relative incidence of AEs was performed in this study to validate the robustness of the model. Second, utility data was extracted from the original model due to a lack of utility data in the Chinese population. There is uncertainty regarding whether the values represent the Chinese population entirely, which also suggests that the Chinese local utilities system of these diseases can be built into further studies, and this can be useful for the development of pharmacoeconomic in China. Lastly, as in the original model, there were also some assumptions in the model which might have increased 
Page 8 of 10

Sun et al. Economic evaluation of imrecoxib and celecoxib

Table 3 Sensitivity results varying age and treatment duration

\begin{tabular}{|c|c|c|}
\hline Start age & Treatment duration (months) & ICER (imrecoxib vs. celecoxib, \$) \\
\hline 55 & 24 & $2,814.97$ \\
\hline \multirow[t]{2}{*}{65} & 6 & $3,164.79$ \\
\hline & 12 & $3,918.35$ \\
\hline
\end{tabular}

ICER, incremental cost-effectiveness ratio.

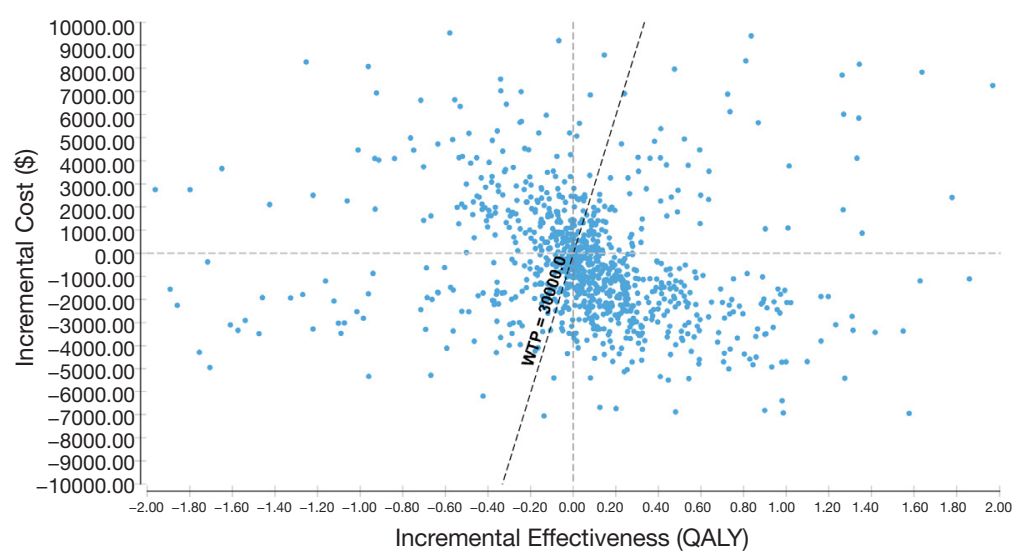

Figure 3 Cost-effectiveness plane. QALYs, quality-adjusted life years.

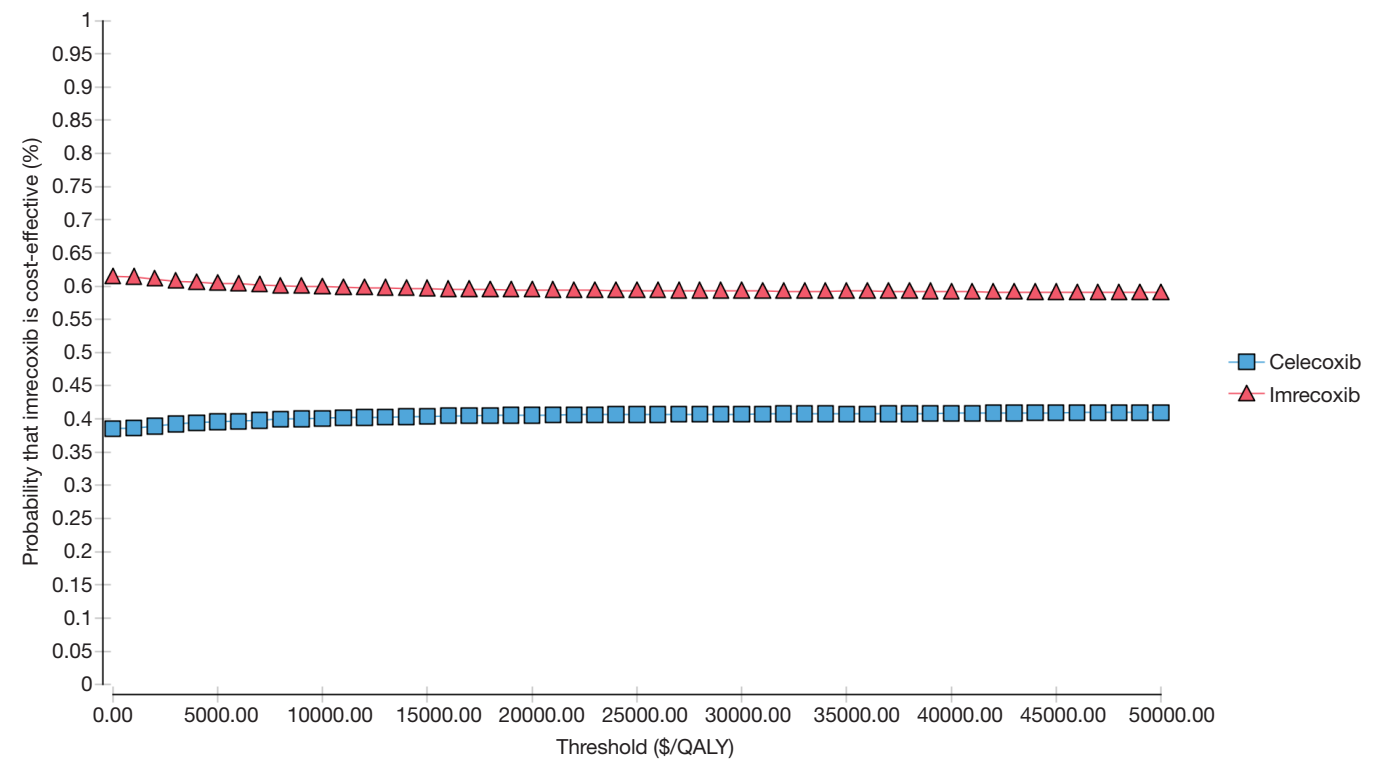

Figure 4 Cost-effectiveness acceptability curve. QALYs, quality-adjusted life years. 
uncertainties in this study. In order to decrease the potential uncertainties caused by these assumptions, both DSA and PSA were carried out.

\section{Conclusions}

Our results showed that, over a lifetime time horizon, although there were uncertainties in this model, treatment with imrecoxib was more cost-effective compared to celecoxib. The key drivers of the model were the relative risk of MI, the cost of imrecoxib, and the utility of OA patients without any AEs. To our knowledge, this study was the first analysis based on real-world data to assess the cost-effectiveness of imrecoxib and celecoxib. These results provide a good reference for relevant stakeholders when managing $\mathrm{OA}$.

\section{Acknowledgments}

We would like to thank Shanghai Su-Value Health Scientific Ltd., Shanghai, China, for their great support with the SuValue Database. We gratefully acknowledge the significant efforts of Shiming Sun and Shaofeng Xue. We also thank Shuyan Gu, Can Zhao, Qilong Gao, and Kaijie Liu for their great help.

Funding: None.

\section{Footnote}

Reporting Checklist: The authors have completed the CHEERS reporting checklist. Available at http://dx.doi. org/10.21037/atm-21-290

Data Sharing Statement: Available at http://dx.doi. org/10.21037/atm-21-290

Conflicts of Interest: All authors have completed the ICMJE uniform disclosure form (available at http://dx.doi. org/10.21037/atm-21-290). The authors have no conflicts of interest to declare.

Ethical Statement: The authors are accountable for all aspects of the work in ensuring that questions related to the accuracy or integrity of any part of the work are appropriately investigated and resolved. This study was approved by the institutional review board of Zhejiang University School of Public Health (No. 20180923). Participants were given informed consent before taking part. All procedures performed in this study involving human participants were in accordance with the Declaration of Helsinki (as revised in 2013).

Open Access Statement: This is an Open Access article distributed in accordance with the Creative Commons Attribution-NonCommercial-NoDerivs 4.0 International License (CC BY-NC-ND 4.0), which permits the noncommercial replication and distribution of the article with the strict proviso that no changes or edits are made and the original work is properly cited (including links to both the formal publication through the relevant DOI and the license). See: https://creativecommons.org/licenses/by-nc-nd/4.0/.

\section{References}

1. Corti MC, Rigon C. Epidemiology of osteoarthritis: prevalence, risk factors and functional impact. Aging Clin Exp Res 2003;15:359-63.

2. Qi L, Yao Y, Jing J. Research progress of matrix metalloproteinase inhibitors in the treatment of osteoarthritis Medical Journal of Qilu 2017;32:111-3.

3. Joint Surgery Group Orthopedics Branch Chinese Medical Association. Guidelines for diagnosis and treatment of osteoarthritis (2018 Edition). Chinese Journal of Orthopaedics 2018;38:705-15.

4. Sun X, Zhen X, Hu X, et al. Osteoarthritis in the MiddleAged and Elderly in China: Prevalence and Influencing Factors. Int J Environ Res Public Health 2019;16:4701.

5. Qinyun X, Kunzheng W, Fuxing P, et al. The survey of the prevalence of primary osteoarthritis in the population aged 40 years and over in China. Chinese Journal of Orthopaedics 2015;35:1206-12.

6. Hong L. The Influence of Aging Population on China's Economy in the Information Society. 2010 2nd IEEE International Conference on Information Management and Engineering; Chengdu, China, 2010:1-4.

7. Jevsevar DS. Treatment of osteoarthritis of the knee: evidence-based guideline, 2nd edition. J Am Acad Orthop Surg 2013;21:571-6.

8. Brereton N, Pennington B, Ekelund M, et al. A costeffectiveness analysis of celecoxib compared with diclofenac in the treatment of pain in osteoarthritis (OA) within the Swedish health system using an adaptation of the NICE OA model. J Med Econ 2014;17:677-84.

9. Dammann HG. Preferential COX-2 inhibition: its clinical relevance for gastrointestinal non-steroidal antiinflammatory rheumatic drug toxicity. Z Gastroenterol 
1999;37:45-58.

10. Zongru G. Development of a new national class 1 drug irexib. Chinese Journal of New Drugs 2012;21:223-30.

11. Chen XH, Bai JY, Shen F, et al. Imrecoxib: a novel and selective cyclooxygenase 2 inhibitor with antiinflammatory effect. Acta Pharmacol Sin 2004;25:927-31.

12. Jianglin H, Shuangan C, Hui S, et al. A Multicenter, Double-Blind and Randomized Controlled Phase II trial of Imrecoxib in Treatment of Knee Osteoarthritis. Chinese Pharmaceutical Journal 2011;46:1740-5.

13. Dong X, Fengchun Z, Jieruo G, et al. A Multicenter Double Blind and Randomized Controlled Phase III Trial of Imrecoxib in the Treatment of Knee Osteoarthritis. Chinese Journal of Allergy \& Clinical Immunology 2014;8:205-10.

14. Beijing Medicine Sunshine Purchasing. Guidelines for drug purchase service. 2019. Available online: https:// yp.bjmbc.org.cn/view/index/guide.html. 2019.

15. Nasef SA, Shaaban AA, Mould-Quevedo J, et al. The cost-effectiveness of celecoxib versus non-steroidal antiinflammatory drugs plus proton-pump inhibitors in the treatment of osteoarthritis in Saudi Arabia. Health Econ Rev 2015;5:53.

16. Brereton N, Winn B, Akehurst R. The cost-effectiveness of celecoxib vs diclofenac in the treatment of osteoarthritis in the UK; an update to the NICE model using data from the CONDOR trial. J Med Econ 2012;15:465-72.

17. National Institute for Health and Care Excellence. Osteoarthritis:Clinical Guideline CG177 Appendices. 2014. Available online: https://www.nice.org.uk/guidance/ cg177/evidence/appendices-pdf-191761310. 2019.

18. Liu G, Hu S, Wu J, et al. China Guidelines for pharmacoeconomic evaluations (Chinese-English version). China Market Press; 2020.

19. Yani P, Wenli P, Zheng X. Effect of nursing on health behavior and recurrence rate of patients with digestive ulcer. Journal of Mathematical Medicine 2016;29:645-7.

20. Xingjiang $W$, Zhongshou $Z$, Xuehao W. Surgical diagnosis and treatment of 317 cases of gastrointestinal hemorrhage.

Cite this article as: Sun X, Zhen X, Hu X, Li Y, Gu S, Gu Y, Zhao Z, Yang W, Dong H. Cost-utility analysis of imrecoxib compared with celecoxib for patients with osteoarthritis. Ann Transl Med 2021;9(7):575. doi: 10.21037/atm-21-290
Journal of Jinling Hospital 1992:231-3.

21. Wang C, Gao Y, Zhu L, et al. Treatment Patterns in Patients With Newly Diagnosed Type 2 Diabetes in China: A Retrospective, Longitudinal Database Study. Clin Ther 2019;41:1440-52.

22. Chinese National Health Commission. 2019 Chinese Health Statistics Yearbook 2019. Peking Union Medical College Press; 2020.

23. Chen H, Zhong B, Li C. Epidemiological investigation of dyspepsia in Guangdong urban residents. Chinese Journal of Internal Medicine 1998;37:312.

24. Li Z, Zou D, Ma X, et al. Epidemiology of peptic ulcer disease: endoscopic results of the systematic investigation of gastrointestinal disease in China. Am J Gastroenterol 2010;105:2570-7.

25. National Bureau of Statistics. Statistical bulletin of national economic and social development of the people's Republic of China in 2019. 2020. Available online: http://www.stats. gov.cn/tjsj/zxfb/202002/t20200228_1728913.html. 2020.

26. Dong H, Buxton M. Early assessment of the likely costeffectiveness of a new technology: A Markov model with probabilistic sensitivity analysis of computer-assisted total knee replacement. Int J Technol Assess Health Care 2006;22:191-202.

27. Delea TE, Karnon J, Smith RE, et al. Cost-effectiveness of extended adjuvant letrozole therapy after 5 years of adjuvant tamoxifen therapy in postmenopausal women with early-stage breast cancer. Am J Manag Care 2006;12:374-86.

28. Claxton K, Sculpher M, McCabe C, et al. Probabilistic sensitivity analysis for NICE technology assessment: not an optional extra. Health Econ 2005;14:339-47.

29. Lu ZH, Xiong XY, Zhang BL, et al. Evaluation of 2 celecoxib derivatives: analgesic effect and selectivity to cyclooxygenase-2/1. Acta Pharmacol Sin 2005;26:1505-11.

30. National Health Commission of the People's Republic of China. List of National Basic Insurance Drugs. 2018. Available online: http://www.nhc.gov.cn/wjw/jbywml/2018 10/600865149f4740eb8ebe729c426fb5d7.shtml. 2020. 
Table S1 Distributional information for model inputs

\begin{tabular}{|c|c|c|c|c|c|c|c|}
\hline \multirow{2}{*}{ Variables name } & \multirow{2}{*}{ Distribution } & \multirow{2}{*}{ Estimate } & \multirow{2}{*}{ a } & \multirow{2}{*}{$\mathrm{b}$} & \multirow{2}{*}{ SE } & \multicolumn{2}{|c|}{$95 \% \mathrm{Cl}$} \\
\hline & & & & & & Lower & Upper \\
\hline Cost-imrecoxib & Gamma & 19.8900 & & & 5.0740 & 9.9450 & 29.8350 \\
\hline Cost-celecoxib & Gamma & 9.3200 & & & 2.3776 & 4.6600 & 13.9800 \\
\hline Cost-topical diclofenac & Gamma & 20.3000 & & & 5.1786 & 10.1500 & 30.4500 \\
\hline Cost-managing $\mathrm{OA}$ without any drugs & Gamma & 26.5000 & & & 6.7602 & 13.2500 & 39.7500 \\
\hline Cost-Gl & Gamma & 10.5200 & & & 2.6837 & 5.2600 & 15.7800 \\
\hline Cost-ulcer & Gamma & 34.5700 & & & 8.8189 & 17.2850 & 51.8550 \\
\hline Cost-complicated Gl events & Gamma & $1,354.1500$ & & & 345.4464 & 677.0750 & 2031.2250 \\
\hline Cost-stroke & Gamma & $1,289.9200$ & & & 329.0612 & 644.9600 & 1934.8800 \\
\hline Cost-MI & Gamma & $5,190.9300$ & & & 1324.2168 & 2595.4650 & 7786.3950 \\
\hline Cost-HF & Gamma & $1,182.3300$ & & & 301.6148 & 591.1650 & 1773.4950 \\
\hline Cost-post ulcer & Gamma & 0.8100 & & & 0.2066 & 0.4050 & 1.2150 \\
\hline Cost-post complicated Gl events & Gamma & 21.5400 & & & 5.4949 & 10.7700 & 32.3100 \\
\hline Cost-post stroke & Gamma & 619.5900 & & & 158.0587 & 309.7950 & 929.3850 \\
\hline Cost-post MI & Gamma & 948.4700 & & & 241.9566 & 474.2350 & 1422.7050 \\
\hline Cost-post HF & Normal & 451.2900 & & & 115.1250 & 225.6450 & 676.9350 \\
\hline Probability-celecoxib-GI & Beta & 0.1245 & 775.0125 & $3,466.9875$ & & 0.0623 & 0.1868 \\
\hline Probability-celecoxib-ulcer & Beta & 0.0009 & 5.6025 & $4,236.3975$ & & 0.0005 & 0.0014 \\
\hline Probability-celecoxib-complicated GI events & Beta & 0.0005 & 3.1125 & $4,238.8875$ & & 0.0003 & 0.0008 \\
\hline Probability-celecoxib-stroke & Beta & 0.0002 & 1.2450 & $4,240.7550$ & & 0.0001 & 0.0003 \\
\hline Probability-celecoxib-MI & Beta & 0.0015 & 9.3375 & $4,232.6625$ & & 0.0008 & 0.0023 \\
\hline Probability-celecoxib-HF & Beta & 0.0004 & 2.4900 & $4,239.5100$ & & 0.0002 & 0.0006 \\
\hline Probability-general-death (age years $=55-64$ ) & Uniform & 0.0037 & & & & 0.0019 & 0.0056 \\
\hline Probability-general-death (age years $=65-74$ ) & Uniform & 0.0094 & & & & 0.0047 & 0.0141 \\
\hline Probability-general-death (age years=75-80) & Uniform & 0.0267 & & & & 0.0134 & 0.0401 \\
\hline Probability-complicated Gl events-death & Uniform & 0.0951 & & & & 0.0476 & 0.1427 \\
\hline Probability-post complicated Gl events-death & Uniform & 0.0277 & & & & 0.0139 & 0.0416 \\
\hline Probability-stroke-death & Uniform & 0.0323 & & & & 0.0162 & 0.0485 \\
\hline Probability-post stroke-death & Uniform & 0.0046 & & & & 0.0023 & 0.0069 \\
\hline Probability-HF-death & Uniform & 0.0495 & & & & 0.0248 & 0.0743 \\
\hline Probability-post HF-death & Uniform & 0.0017 & & & & 0.0009 & 0.0026 \\
\hline Probability-MI-death & Uniform & 0.0428 & & & & 0.0214 & 0.0642 \\
\hline Probability-post MI-death & Uniform & 0.0045 & & & & 0.0023 & 0.0068 \\
\hline Probability-COX-2-withdraw & Beta & 0.1120 & 446.5440 & $3,540.4560$ & & 0.0560 & 0.1680 \\
\hline Utility-OA patients with treatment of COX-2 & Beta & 0.1806 & & & 0.0461 & 0.0903 & 0.2709 \\
\hline Relative risk-Gl-imrecoxib vs. celecoxib & Lognormal & 0.4900 & & & & 0.2450 & 0.7350 \\
\hline Relative risk-ulcer-imrecoxib vs. celecoxib & Lognormal & 1.3400 & & & & 0.6700 & 2.0100 \\
\hline Relative risk-complicated Gl events-imrecoxib vs. celecoxib & Lognormal & 0.5000 & & & & 0.2500 & 0.7500 \\
\hline Relative risk-stroke-imrecoxib vs. celecoxib & Lognormal & 1.0000 & & & & 0.5000 & 1.5000 \\
\hline Relative risk-MI-imrecoxib vs. celecoxib & Lognormal & 1.0000 & & & & 0.5000 & 1.5000 \\
\hline Relative risk-HF-imrecoxib vs. celecoxib & Lognormal & 1.0000 & & & & 0.5000 & 1.5000 \\
\hline Relative risk-utility-GI & Lognormal & 0.7330 & & & & 0.3665 & 1.0995 \\
\hline Relative risk-utility-withdraw due to $\mathrm{Gl}$ & Lognormal & 0.9890 & & & & 0.4945 & 1.4835 \\
\hline Relative risk-utility-ulcer & Lognormal & 0.5520 & & & & 0.2760 & 0.8280 \\
\hline Relative risk-utility-post ulcer & Lognormal & 0.9800 & & & & 0.4900 & 1.4700 \\
\hline Relative risk-utility-complicated Gl events & Lognormal & 0.4590 & & & & 0.2295 & 0.6885 \\
\hline Relative risk-utility-post complicated GI events & Lognormal & 0.9800 & & & & 0.4900 & 1.4700 \\
\hline Relative risk-utility-stroke & Lognormal & 0.3480 & & & & 0.1740 & 0.5220 \\
\hline Relative risk-utility-post stroke & Lognormal & 0.7060 & & & & 0.3530 & 1.0590 \\
\hline Relative risk-utility-MI & Lognormal & 0.3740 & & & & 0.1870 & 0.5610 \\
\hline Relative risk-utility-post MI & Lognormal & 0.8800 & & & & 0.4400 & 1.3200 \\
\hline Relative risk-utility-HF & Lognormal & 0.7100 & & & & 0.3550 & 1.0650 \\
\hline Relative risk-utility-post HF & Lognormal & 1.0000 & & & & 0.5000 & 1.5000 \\
\hline Relative risk-utility-post treatment & Lognormal & 0.9890 & & & & 0.4945 & 1.4835 \\
\hline Relative risk-risk of bleeding/ulcer (aged 65) vs. (aged 55) & Lognormal & 2.9600 & & & & 1.4800 & 4.4400 \\
\hline Relative risk-risk of CV (aged 65) vs. (aged 55) & Lognormal & 1.9400 & & & & 0.9700 & 2.9100 \\
\hline
\end{tabular}

$\mathrm{OA}$, osteoarthritis; COX-2, cyclooxygenase; GI, gastrointestinal; HF, heart failure; Ml, myocardial infarction; CV, cardiovascular diseases. 


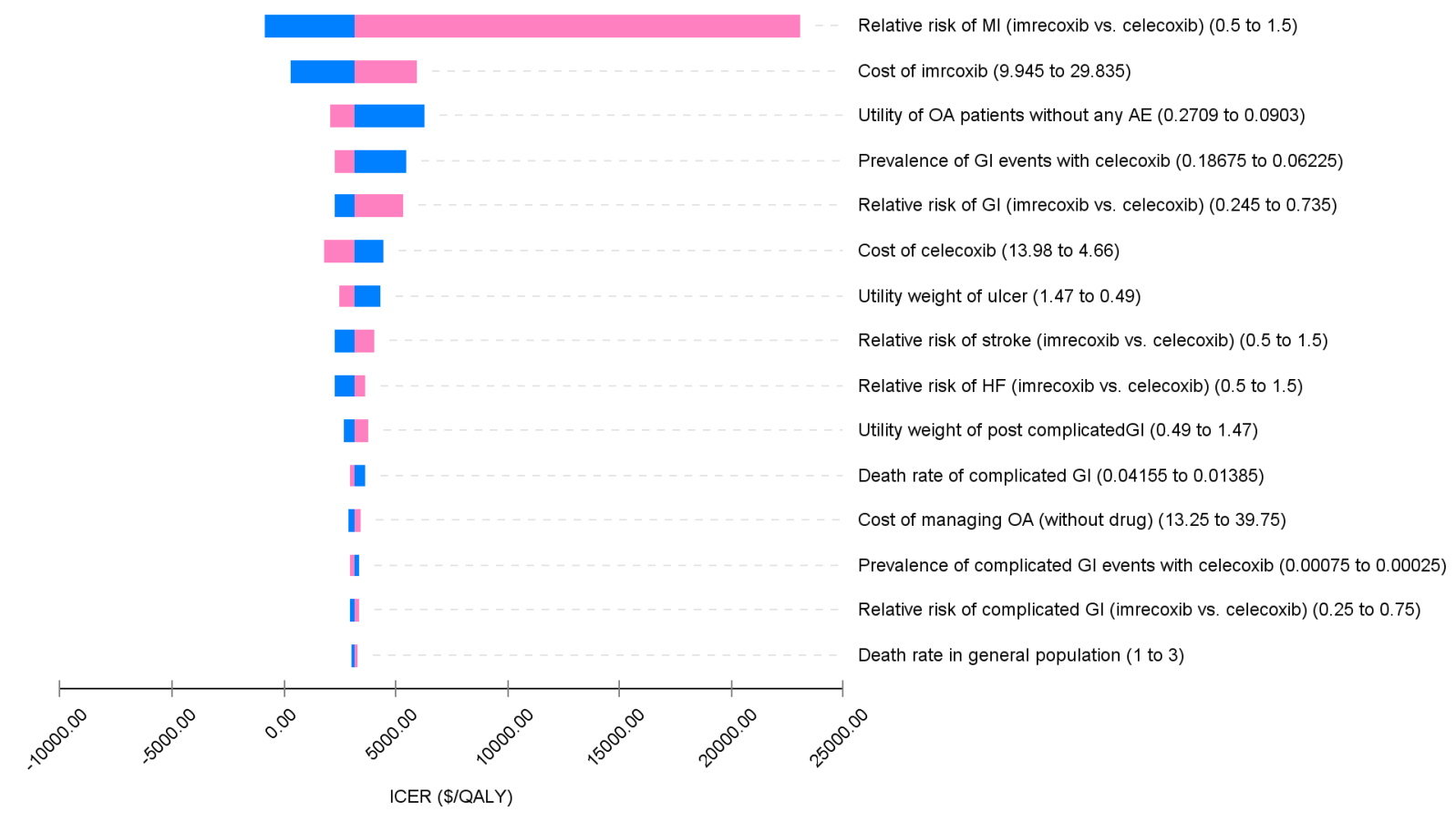

Figure S1 The tornado diagram (imrecoxib versus celecoxib). OA, osteoarthritis; GI, gastrointestinal; HF, heart failure; MI, myocardial infarction. 